\title{
CONGENITAL ABSENCE OF THE PERICARDIUM
}

\author{
HYUN-JIN KIM, MD ${ }^{1,2}$, YOUNG-SEOK CHO, MD ${ }^{1,2}$, GOO-YEONG CHO, MD ${ }^{1,2}$, AND SANG IL CHOI, MD ${ }^{1,3}$ \\ ${ }^{1}$ CARDIovascular CENTER, SEOUl NATIONAL UNIVERSITY BUNDANG HOSPITAL, SEONGNAM, KOREA \\ 2DEPARTMENT OF INTERNAL MEDICINE, SEOUL NATIONAL UNIVERSITY COLLEGE OF MEDICINE, SEOUL, KOREA \\ ${ }^{3}$ DEPARTMENT OF RADIOLOGY, SEOUL NATIONAL UNIVERSITY BUNDANG HOSPITAL, SEONGNAM, KOREA
}

Congenital absence of the pericardium is a rare cardiac malformation and is most often asymptomatic. It is usually discovered as an incidental finding. Physical examination, chest radiography, and electrocardiogram are often unremarkable. Echocardiography provides valuable information, and sometimes computed tomography or magnetic resonance imaging is needed for subsequent confirmation.

KEY WORDS: Absence of the pericardium - Complete defect · Partial defect.

\section{INTRODUCTION}

Congenital absence of the pericardium is a rare cardiac malformation. ${ }^{1)}$ Defective development of the pleuropericardial membranes, which fuse at the midline and separate the pericardial and pleural cavities, causes complete or partial absence of the pericardium and partial defect is more common than complete defect. ${ }^{2)}$ Patients with absence of the pericardium are most often asymptomatic, but they sometimes present with various clinical manifestations. ${ }^{3)}$ Herein, we report three cases of congenital absence of the pericardium. Two cases involve absence of the left pericardium, and the other case is complete absence of the pericardium.

\section{CASE}

A 19-year-old woman was referred to our clinic for further evaluation of cardiomegaly on chest radiography. She had no chest pain, shortness of breath, or other specific signs and symptoms. Her past medical history and family history were unremarkable. She had not taken any medications and denied use of illicit drugs and tobacco or alcohol abuse. On cardiac examination, a regular heart rhythm was heard without heart murmur. A 12-lead electrocardiogram (ECG) showed normal sinus rhythm, with a heart rate of $62 \mathrm{bpm}$ and right deviation of the heart axis (Fig. 1A). The chest radiograph revealed that the heart had bulging contour of the left superior cardiac border and leftward shift (Fig. 1B). The echocardiography showed a globe-shaped heart and bulbous ventricle due to suspension of the heart from its basal pedicle (Fig. 1C). It also revealed mitral valve prolapse. The estimated left ventricular ejection fraction was $65 \%$. Chest computed tomography (CT) revealed outpouching of the right ventricle (RV), which contacted the left chest wall, and interposition of the lung between the aortic arch and prominent pulmonary trunk (Fig. 1D and E). The patient was diagnosed with congenital absence of the left pericardium.

A 56-year-old man was referred to our clinic for ECG abnormality. He had no chest pain, dyspnea, or other specific signs and symptoms. His past medical history and family history were unremarkable. He neither took medications nor used tobacco or alcohol. On examination, his blood pressure was $126 / 76 \mathrm{mmHg}$. A systolic ejection-type murmur was present with regular heart rhythm. A 12-lead ECG showed sinus rhythm with a heart rate of $59 \mathrm{bpm}$, an incomplete right bundle branch block, and a slight increase in left ventricular voltage (Fig. 2A). Chest radiography revealed flattening of the left heart border and a lucent area between the aorta and pulmonary artery due to lung interposition (Fig. 2B). The patient underwent an echocardiogram and chest CT because of the abnormal chest radiography, murmur, and ECG. The echocardiography showed a left laterally displaced left ventricle (LV) apex with a swinging motion that was highly suggestive of congenital absence of the pericardium (Fig. 2C). The RV cavity was enlarged as $38 \mathrm{~mm}$ and demonstrated paradoxical septal motion. LV systolic function was normal. The chest CT re-

- Received: August 16, 2013 •Revised: December 2, 2013 •Accepted: February 18, 2014

- Address for Correspondence: Young-Seok Cho, Division of Cardiology, Department of Internal Medicine, Seoul National University College of Medicine,

82 Gumi-ro 173beon-gil, Bundang-gu, Seongnam 463-707, Korea Tel: +82-31-787-7018, Fax: +82-31-787-4290, E-mail: flammeus1@gmail.com

- This is an Open Access article distributed under the terms of the Creative Commons Attribution Non-Commercial License (http://creativecommons.org/licenses/by-nc/3.0)

which permits unrestricted non-commercial use, distribution, and reproduction in any medium, provided the original work is properly cited. 

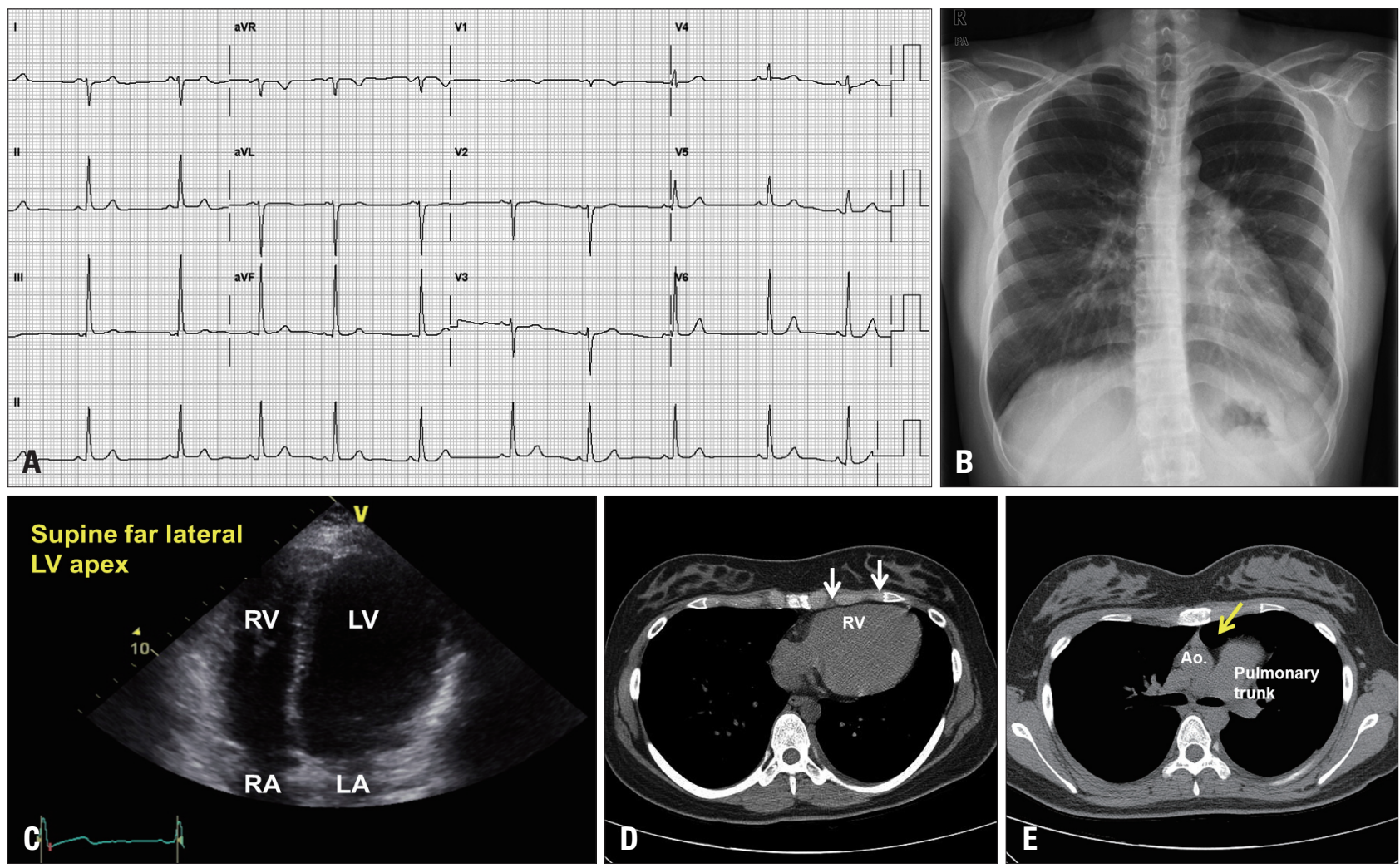

Fig. 1. A: 12-lead electrocardiogram demonstrating normal sinus rhythm and right deviation of the heart axis. B: Chest radiograph demonstrating leftward position of the heart and the bulging contour of the left heart border. C: Echocardiography apical four chamber view: echocardiography showed a globe-shaped heart and bulbous ventricle. D: Chest CT revealed outpouching of the RV, which contacted the left chest wall (white arrows). $\mathrm{E}$ : Chest CT showed interposition of the lung between the aortic arch and pulmonary trunk (yellow arrow). LA: left atrium, LV: left ventricle, RA: right atrium, RV: right ventricle, Ao: aorta.

vealed nonvisualization of the pericardium and leftward displacement of the entire heart with mild RV dilatation (Fig. 2D). The patient was diagnosed with congenital absence of the pericardium.

A 71-year-old male patient presented with a history of dyspnea on exertion. He reported atypical chest pain and shortness of breath. He suffered from asthma and was taking medication. Medical and family histories were otherwise unremarkable. He was a current, 100 pack-year smoker. On examination, his blood pressure was 120/70 mmHg. Regular heart rhythm without murmur and clear breath sounds were heard on auscultation. A 12-lead ECG showed normal sinus rhythm, with a heart rate of $73 \mathrm{bpm}$ and left deviation of the heart axis (Fig. 3A). Q-waves were seen in leads II, aVF, V1, V2, and V3. Chest radiography showed bulging contour of the left superior cardiac border (Fig. 3B). Due to his symptoms, abnormal chest radiography, and ECG, the patient underwent an echocardiogram and heart CT. The echocardiography revealed a laterally displaced LV apex that was highly suggestive of congenital absence of the pericardium (Fig. 3C). It also showed an enlarged LV cavity with mild global hypokinesia without regional wall motion abnormality. Valvular morphology and function were normal, and the estimated LV ejection fraction was $47 \%$. The cardiac CT revealed absence of the pericardium at the left side of the heart without evidence of associated anomaly such as atrial septal defect or ventricular septal defect (Fig. $3 \mathrm{D})$. The patient was diagnosed with congenital absence of the left pericardium.

\section{DISCUSSION}

Congenital absence of the pericardium is a rare malformation in which the fibroserous membrane covering the heart is absent. Insufficient blood supply to the pleuropericardium owing to premature atrophy of the left common cardiac vein leads to defective development of the pleuropericardial membranes, and results in pericardial deficiencies. ${ }^{2)}$ The most common defect is absence of the left pericardium (70\%), with a male preponderance. ${ }^{1)}$ Complete absence of the pericardium (9\%) and absence of the right pericardium (17\%) are fewer than the absence of the left pericardium. ${ }^{2)}$

Although patients with congenital absence of the pericardium usually have isolated defects, almost $30 \%$ of these patients have other congenital cardiovascular or pulmonary anomalies. ${ }^{4)}$ These patients also may have other syndromes like VATER syndrome (vertebral defects, anal atresia, tracheoesophageal fistula, and radial and renal dysplasia), ${ }^{5)}$ Marfan's syndrome, and Pallister-Killian syndrome (tetrasomy $12 \mathrm{p}$ ). ${ }^{677}$

Congenital absence of the pericardium is usually asymptom- 

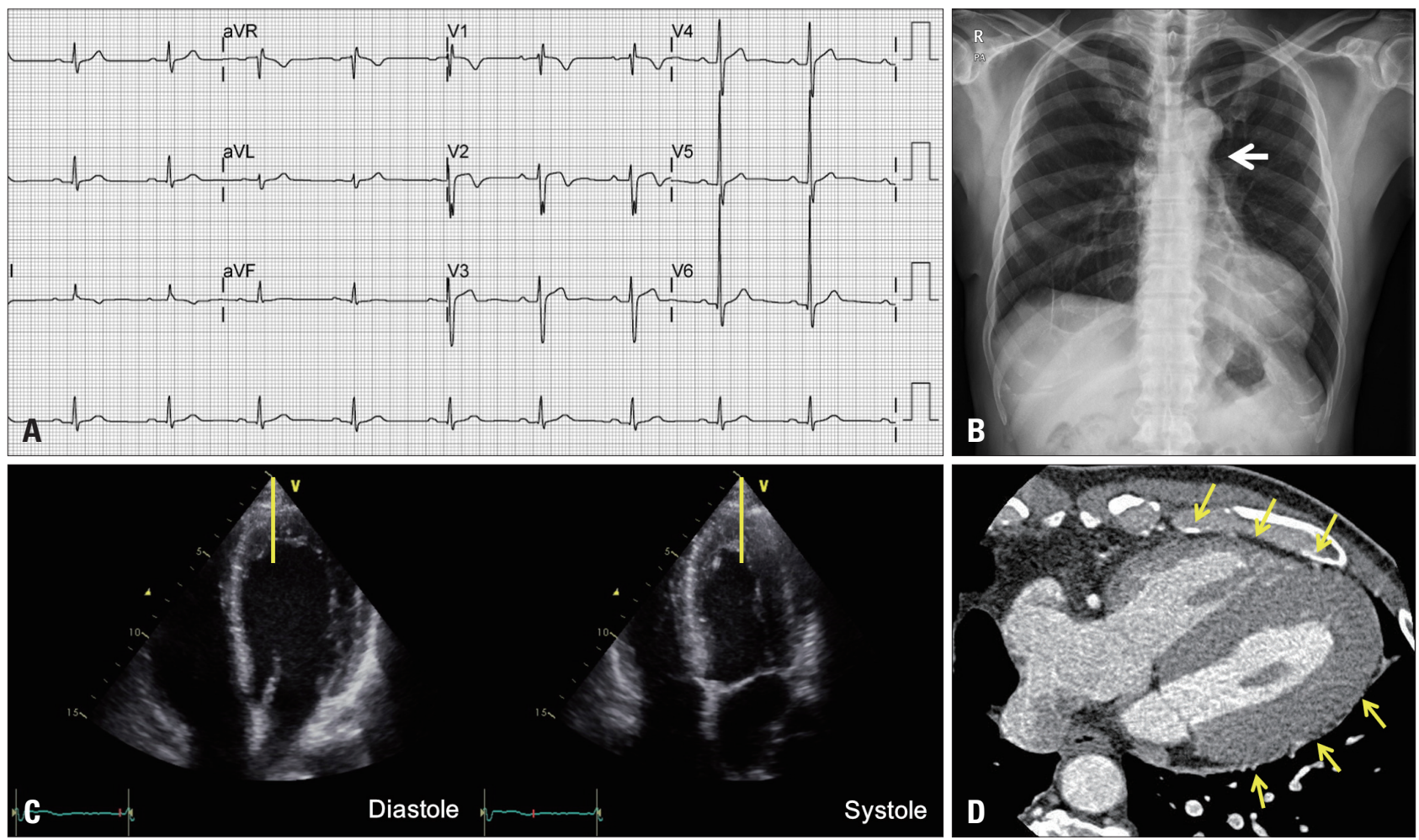

Fig. 2. A: 12-lead electrocardiogram demonstrating sinus rhythm with an incomplete right bundle branch block. B: Chest radiograph demonstrating leftward position of the heart, flattening of the left heart border, and a lucent area between the aorta and pulmonary artery (white arrow). C: Echocardiography apical four chamber view: left ventricular apex showed a swinging motion in diastole and systole. D: Chest CT revealed nonvisualization of the pericardium (yellow arrows) and left-ward displacement of the entire heart with mild right ventricular dilatation.
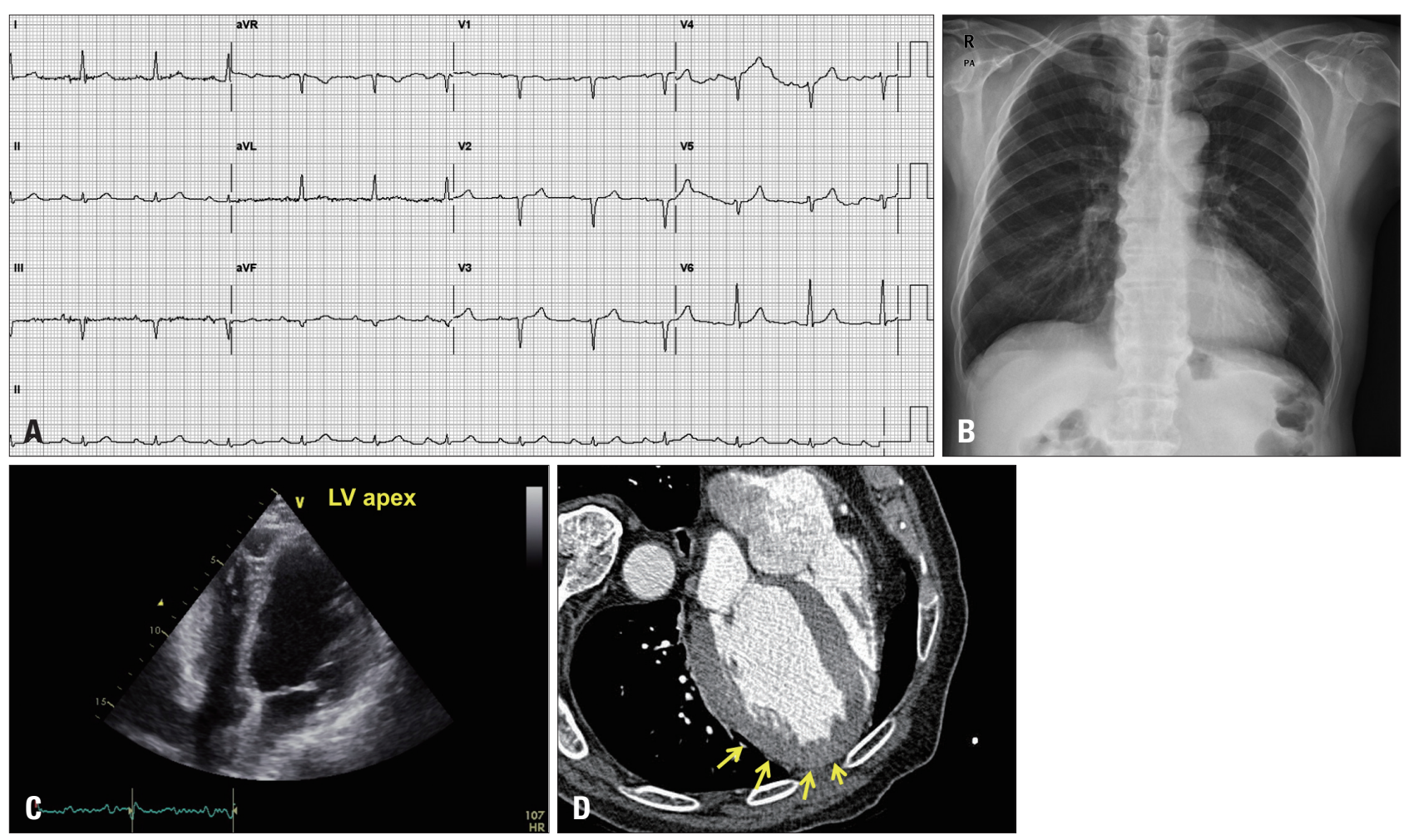

Fig. 3. A: Electrocardiogram demonstrating normal sinus rhythm with left deviation of the heart axis. B: Bulging contour of the left superior cardiac border. C: Apical four chamber view: echocardiography showed a laterally displaced left ventricular apex. D: The cardiac CT revealed absence of the pericardium at the left side of the heart (yellow arrows). 
atic. However, it can be detected with various clinical manifestations like non-exertional chest pain of lancinating nature, dyspnea, dizziness, palpitations. ${ }^{2)}$ Usual chest radiography findings include marked leftward displacement of the cardiac silhouette without tracheal deviation and the flattened and elongated left ventricular border, as was seen in the cases described. A prominent pulmonary artery and interposed lung tissue cause a lucent area between the diaphragm and heart or aorta and pulmonary artery. ${ }^{8)}$ The ECG typically reveals bradycardia with right bundle branch block. Also, poor R wave progression and prominent $\mathrm{P}$ waves could be observed. ${ }^{1) 8}$ Right bundle branch block can be seen on ECG, as in our patient.

Echocardiography could support the diagnosis and suggest CT and magnetic resonance imaging (MRI) examinations as further confirmatory tests. In congenital absence of the pericardium, a more lateral and more superior echocardiographic window is necessary. Also, paradoxical or flat systolic motion of the ventricular septum with normal systolic thickening, cardiac hypermobility, and an enlarged RV during systole can be seen. ${ }^{2)}$ In addition, the 'teardrop' appearance of the LV, bulbous ventricle, and elongated atria in the apical four chamber view can be seen on echocardiography. Lack of fixation of the heart in the mediastinum appears to cause that finding. ${ }^{122}$ Since confirmation of the diagnosis and the distinction between partial and complete defects are clinically important, other imaging tests are required. CT and MRI scans can distinguish between complete and partial defects. CT and MRI can show herniation of the heart through the partial defects of pericardium. ${ }^{910)}$ Rightward axis shift can be seen on decubitus CT in complete defects, also the attachment of the right pericardium to the anterior chest wall, interposition of the left lung tissue between main pulmonary artery and aorta, and prominent main pulmonary artery can be seen on CT. ${ }^{10) 11 \text { ) }}$ These CT findings were demonstrated in our cases.

Patients with complete defect of pericardium usually have excellent prognosis. However, in patients with partial absence, herniation of the heart through the partial defect of pericardium can lead to tricuspid regurgitation by traction of the chordal structures, fatal myocardial strangulation, ischemia, and sudden death. ${ }^{112)}$ Small defects or complete defects, as in our cases, do not usually need any intervention unless complications occur. ${ }^{2) 13)}$ On the other hand, when herniation occurs or is imminent in partial defects, surgical widening of the defect by pericardiectomy or pericardioplasty may be performed. ${ }^{1)}$ Indi- cation for surgical intervention in patients with partial absence of the pericardium is mainly determined by the symptoms, size, and location of the defect. ${ }^{2)}$

Pericardial agenesis is a rare cardiac malformation and there are no pathognomonic features on clinical presentation, ECG, or chest radiography. Therefore, echocardiography, CT, and MRI provide valuable information that can diagnose the presence of partial and complete pericardial agenesis. Complete defect cases usually show an excellent prognosis and need surgical treatment only in cases of complications or intractable symptoms.

\section{REFERENCES}

1. Abbas AE, Appleton CP, Liu PT, Sweeney JP. Congenital absence of the pericardium: case presentation and review of literature. Int J Cardiol 2005;98:21-5.

2. Centola M, Longo M, De Marco F, Cremonesi G, Marconi M, Danzi GB. Does echocardiography play a role in the clinical diagnosis of congenital absence of pericardium? A case presentation and a systematic review. $J$ Cardiovasc Med (Hagerstown) 2009;10:687-92.

3. D'Altorio R, Cano JY. Congenital abscence of the left pericardium detected by imaging of the lung: case report. J Nucl Med 1977;18:267-8.

4. Eyileten Z, Arikbuka M, Yazicioğlu L, Ozyurda U. Left pericardial agenesis in a patient with sinus venosus type atrial septal defect. Anadolu Kardiyol Derg 2007;7:205-6.

5. Lu C, Ridker PM. Echocardiographic diagnosis of congenital absence of the pericardium in a patient with VATER association defects. Clin Cardiol 1994:17:503-4.

6. Zakowski MF, Wright Y, Ricci A Jr. Pericardial agenesis and focal aplasia cutis in tetrasomy 12p (Pallister-Killian syndrome). Am J Med Genet 1992;42:323-5.

7. Boscherini B, Galasso C, Bitti ML. Abnormal face, congenital absence of the left pericardium, mental retardation, and growth hormone deficiency. Am J Med Genet 1994:49:111-3.

8. Sivrikoz MC, Durceylan E, Boztepe H, Birdane A. Congenital total absence of pericardium in a patient with left lung lower lobe bronchiectasis. Anadolu Kardiyol Derg 2011;11:81-3.

9. Lau KW, Ding ZP. Images in cardiovascular medicine. Partial pericardial defect. Circulation 1998;97:1992.

10. Faridah Y, Julsrud PR. Congenital absence of pericardium revisited. Int J Cardiovasc Imaging 2002;18:67-73.

11. Hoey ET, Yap KS, Darby MJ, Mankad K, Puppala S, Sivananthan MU. Complete left pericardial defect: evaluation with supine and decubitus dual source CT. J Cardiovasc Comput Tomogr 2009;3:417-9.

12. Hotouras A, Shahin Y. Pericardial agenesis: a rare cause of chest pain. Postgrad Med J 2010;86:380-1.

13. Gatzoulis MA, Munk MD, Merchant N, Van Arsdell GS, McCrindle BW, Webb GD. Isolated congenital absence of the pericardium: clinical presentation, diagnosis, and management. Ann Thorac Surg 2000;69: 1209-15. 